\title{
Mechanics of wedge turns in alpine skiing
}

Authors: Serguei Komissarov, School of Mathematics, University of Leeds, Leeds, LS29JT, UK. email: s.s.komissarov@leeds.ac.uk.

Status: preprint.

Preprint DOI: 10.31236/osf.io/qv6j5

Cite as: Komissarov, Serguei. 2020. "Mechanics of Wedge Turns in Alpine Skiing." SportRxiv. July 12. doi:10.31236/osf.io/qv6j5. 


\title{
Mechanics of wedge turns in alpine skiing
}

\author{
Serguei S. Komissarov \\ Department of Applied Mathematics \\ The University of Leeds \\ Leeds, LS2 9JT, UK \\ email: s.s.komissarov@leeds.ac.uk
}

\begin{abstract}
A simple approximate theory of snow machining is applied to modelling successive wedge turns of alpine skiing. The results are in agreement with available experimental investigations of such turns. In particular, the model explains the abnormally high values for the coefficient of friction reported in these studies.
\end{abstract}

Keywords: alpine skiing, modelling, balance/stability, performance

\section{Introduction}

A wedge turn is the very first type of turns introduced to students in most schools of alpine skiing. In this turn, skis form a wedge pointing in the direction of motion. This greatly increases the braking power of skis and hence allows to keep the speed of descent as low as needed for the students to feel safe and able to focus on improving their technique. The turning action is achieved by preferential loading one of the skis, which leads to turning in the opposite direction. For example, if the skier needs to turn right, then the left ski has to be loaded more than the right ski .

During a wedge turn, both skis are set at an angle (which is called the angle of attack) to the direction of motion. As a result, the motion of each ski is a combination of motions along the ski longitudinal axis and perpendicular to it. The perpendicular motion is particularly important in the mechanics of wedge turns as it leads to removal of a top layer of snow. For this reason, this turn is also known as a snow-plow turn. This snow-removal action is similar to the widely used in manufacturing process of machining (of various materials). In this process, the cutting tool is subject to a reaction force from the machined material, which can be considered as a combination of friction and pressure. The pressure force arises mostly at the rake face of the tool, where it pushes the chip out. Its component tangent to the machined surface is called the cutting force. 
In skiing, the role of the rake face is played by the ski base and hence the cutting force is perpendicular to the ski. It has a component which is opposed to the direction of motion and hence promotes braking. But it also has a component which is perpendicular to the direction of motion, and this component is the reason behind the turning action of skis set at non-vanishing angle of attack.

So far, there have been only a rather limited number of studies of snow machining and attempts to apply it in the theory of ski turns. Lieu and Mote (1984) carried out an experimental study of orthogonal ice cutting at rake angles relevant to skiing (negative rake angles). Later they arrived with empirical expressions for the ice-reaction forces emerging in the interaction, where these forces are described as functions of the rake angle and ice cutting (penetration) depth, and used a scaled version of these functions to model ski-snow interaction in skiing turns (Lieu \& Mote, 1985). A similar study of ice machining, including the oblique case, was carried out by Tada and Hirano (1999) who found a reasonably good agreement with the results of Lieu and Mote (1984).

Tada and Hirano (2002) and Tada and Kobayashi (2005) carried out an experimental study of both orthogonal and oblique machining of compacted snow. In the orthogonal case the relative velocity is orthogonal to the ski longitudinal axis, whereas in the oblique case the angle between the two, the angle of attack, is below $90^{\circ}$. Their results could not be fitted by the functions of Lieu and Mote (1985), especially at small edge angles, so they come up with their own rather complicated empirical expressions for the snow reaction forces and used them to model single skiing turns.

In contrast to these experimental studies, Brown (2009) proposed to use the theory of metal cutting developed by Merchant (1945) for the case of continuous (type 2) chip. However, snow and ice are highly brittle materials and instead of continuous chip their machining normally results in a spray of ice particles.

In our previous work, we developed an approximate theory of snow machining and applied it to some of the most basic manoeuvres of alpine skiing, side-slipping down the fall line and diagonally across the ski slope (Komissarov, 2020). The main simplification of the theory is its neglecting of the Coulomb friction between the ski and the cut snow and its main advantage is the very simple analytical expressions for the turning and braking components of the cutting force. Its application to the side-slipping allowed to explain the abnormally high values of the friction coefficient obtained in the experimental studies of traversing by Kaps, Nachbauer, and Mössner (1996). The theory attributes them not to the usual friction but to the braking component of the snow cutting force.

Similarly high and even higher values for the coefficient of friction were obtained by Sahashi and Ichino (1998) in their investigation of skidded ski turns. Among several different types of such turns, they explored the wedge turn. In fact, this seems to be the only published study of wedge turns so far. Here we hypothesise that this abnormally high "friction" has the same origin as in the basic side-slipping. In 


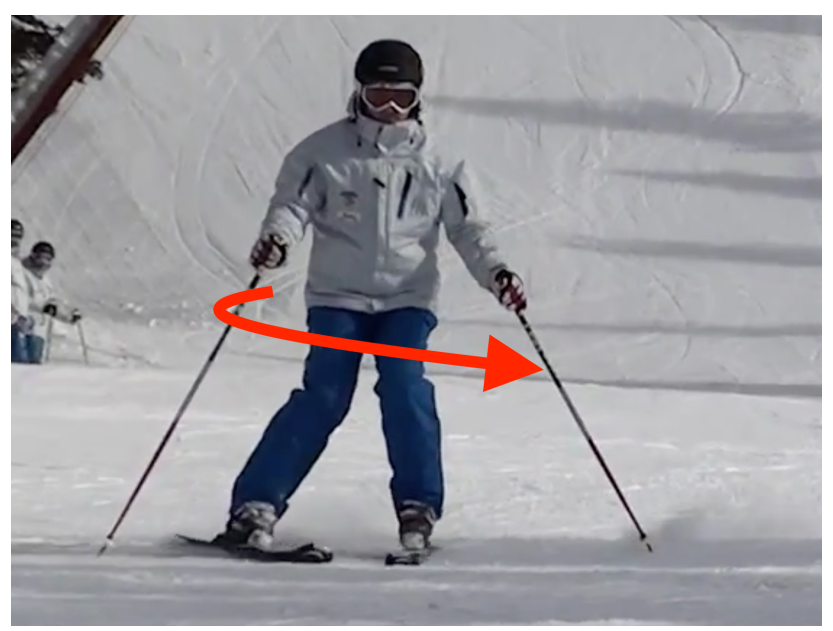

Figure 1. Skier executing left wedge turn. The red line indicates the trajectory of motion.

order to test this, we attempt to reproduce the observed kinematics of wedge turns in simulations based on the theory of snow machining.

\section{Method}

The key forces acting on a skier during a ski run are the gravity force, the snow friction, the snow reaction force, and the aerodynamic drag force. The usual snow friction is normally very low, thanks to the slippery nature of ice, meltwater lubrication, and waxes. As we think that the braking action due to the snow cutting component of the snow reaction force is much more important, we will ignore the usual friction. The aerodynamic drag force strongly depends on skier's speed and becomes important only in high-speed skiing typical for super-G and downhill racing. In contrast, wedge turns are executed only at a rather low speed, and hence we may ignore the aerodynamic drag as well. As the result of these simplifications, the equation of motion reduces to

$$
M \frac{d \boldsymbol{v}}{d t}=M \boldsymbol{g}+\boldsymbol{F}_{\mathrm{r}} .
$$

where $M$ is the skier mass, $\boldsymbol{v}$ is the skiers velocity, $\boldsymbol{g}$ is the gravitational acceleration vector, and $\boldsymbol{F}_{\mathrm{r}}$ is the snow reaction force. Accounting for the contributions from both skis, we write

$$
\boldsymbol{F}_{\mathrm{r}}=\boldsymbol{F}_{\mathrm{r}}^{(l)}+\boldsymbol{F}_{\mathrm{r}}^{(r)},
$$

where "(l)" and "(r)" stand for the left and the right skis respectively. According to our theory of snow machining,

$$
\boldsymbol{F}_{\mathrm{r}}^{(i)}=N^{(i)} \tan \Phi^{(i)} \hat{\boldsymbol{n}}_{\mathrm{s}}^{(i)}+\boldsymbol{N}^{(i)}
$$




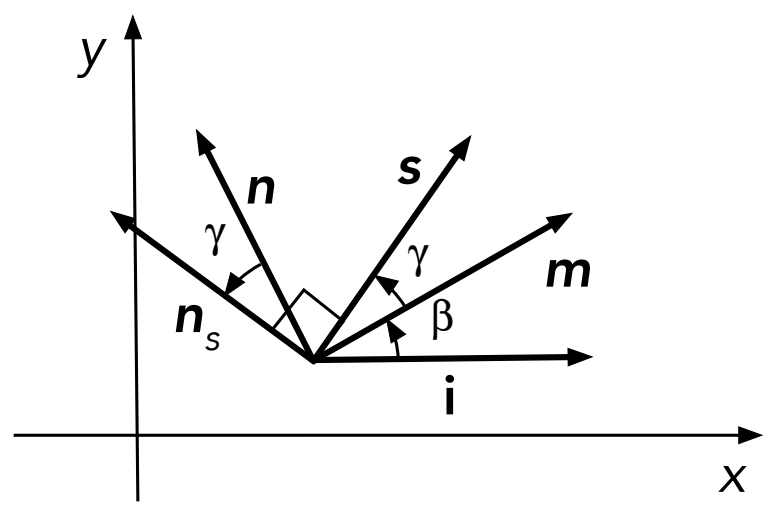

Figure 2. Key unit vectors and angles in the plane of the ski slope. $\hat{\boldsymbol{i}}$ points in the direction of the fall line, $\hat{\boldsymbol{m}}$ in the direction of motion, $\hat{\boldsymbol{s}}$ along the ski axis, $\hat{\boldsymbol{n}}$ is normal to $\hat{\boldsymbol{m}}$, and $\hat{\boldsymbol{n}}_{\mathrm{s}}$ is normal to $\hat{\boldsymbol{s}}$.

where $\boldsymbol{N}^{(i)}=N^{(i)} \hat{\boldsymbol{k}}$ is the normal component of the snow reaction force, $\hat{\boldsymbol{k}}$ is the outgoing unit vector normal to the plane of the ski slope, $\hat{\boldsymbol{n}}_{\mathrm{s}}^{(i)}$ is the unit vector in the plane of the slope which is normal to the edge of the $i$-th ski and points to the side opposite to the direction of motion, and $\Phi^{(i)}$ is the inclination angle of the line connecting the midpoint of $i$-th ski to skier's centre of mass (Komissarov, 2020). For simplicity, we assume that both the skis and the CM move with the same velocity and denote as $\hat{\boldsymbol{m}}$ the unit vector in the direction of motion. Hence

$$
\hat{\boldsymbol{n}}_{\mathrm{s}}^{(i)}=\frac{\left(\hat{\boldsymbol{m}} \times \hat{\boldsymbol{s}}^{(i)}\right) \times \hat{\boldsymbol{s}}^{(i)}}{\left|\hat{\boldsymbol{m}} \times \hat{\boldsymbol{s}}^{(i)}\right|},
$$

where $\hat{\boldsymbol{s}}^{(i)}$ is the unit vector aligned with the $i$-th ski. Using Cartesian coordinates with the basis vectors $\hat{\boldsymbol{k}}$ (normal to the ski slope), $\hat{\boldsymbol{i}}$ (parallel to the fall line), and $\hat{\boldsymbol{j}}$ (perpendicular to the other two), we can write

$$
\begin{aligned}
\boldsymbol{g} & =g \sin \alpha \hat{\boldsymbol{i}}-g \cos \alpha \hat{\boldsymbol{k}} \\
\hat{\boldsymbol{m}} & =\cos \beta \hat{\boldsymbol{i}}+\sin \beta \hat{\boldsymbol{j}} \\
\hat{\boldsymbol{s}} & =\cos (\beta+\gamma) \hat{\boldsymbol{i}}+\sin (\beta+\gamma) \hat{\boldsymbol{j}} \\
\hat{\boldsymbol{n}}_{\mathrm{s}} & =\operatorname{sign}(\gamma)(-\sin (\gamma+\beta) \hat{\boldsymbol{i}}+\cos (\gamma+\beta) \hat{\boldsymbol{j}})
\end{aligned}
$$

where $\alpha$ is the slope inclination angle, $\beta$ is the angle of traverse, and $\gamma$ is the angle of attack. Here we assume that both $\gamma$ and $\beta$ are measured in the counter-clockwise direction as seen from above the slope. The reference direction for $\beta$ is $\hat{j}$ and the reference direction for $\gamma$ is $\hat{\boldsymbol{m}}$.

If we ignore the up-and-down motion of skier's CM, then $\hat{\boldsymbol{k}} \cdot d \boldsymbol{v} / d t=0$, and 
projecting equation (1) on the direction of $\hat{\boldsymbol{k}}$ we find

$$
N=N^{(l)}+N^{(r)}=M g \cos \alpha,
$$

where $N$ is the total normal to the slope component of the snow reaction force, or the total load of the skis. Introducing $A_{N}^{(i)}=N^{(i)} / N$, the load distribution factor of the $i$-th ski, we can write

$$
N^{(i)}=A_{N}^{(i)} M g \cos \alpha .
$$

By definition, $0 \leq A_{N}^{(i)} \leq 1$ and $A_{N}^{(l)}+A_{N}^{(r)}=1$.

Projecting equation (1) onto the slope plane, we obtain

$$
\frac{1}{g} \frac{d \boldsymbol{v}}{d t}=\sin \alpha \hat{\boldsymbol{i}}+\cos \alpha \sum_{i=l, r} A_{N}^{(i)} \tan \Phi^{(i)} \hat{\boldsymbol{n}}_{\mathrm{s}}^{(i)} .
$$

Scalar multiplication of this equation with $\hat{\boldsymbol{m}}$ yields the evolution equation for the skier speed

$$
\frac{1}{g} \frac{d V}{d t}=\sin \alpha \cos \beta-\cos \alpha \sum_{i=l, r} A_{N}^{(i)}\left|\sin \gamma^{(i)}\right| \tan \Phi^{(i)} .
$$

One can also use equation (1) to find the evolution equation for the angle of traverse. To this end, we first note that

$$
V \frac{d \hat{\boldsymbol{m}}}{d t}=\frac{d \boldsymbol{v}}{d t}-\hat{\boldsymbol{m}} \frac{d V}{d t} .
$$

Substituting into this equation the above expressions for $d \boldsymbol{v} / d t$ and $d V / d t$ and projecting the result onto the direction of $\hat{\boldsymbol{i}}$ we obtain

$$
\frac{V}{g} \frac{d \beta}{d t}=-\sin \alpha \sin \beta+\cos \alpha \sum_{i=l, r} A_{N}^{(i)} \operatorname{sgn} \gamma^{(i)} \cos \gamma^{(i)} \tan \Phi^{(i)} .
$$

Once, $V(t)$ and $\beta(t)$ are know, we can find the skier trajectory via integrating

$$
\frac{d x}{d t}=V \cos \beta,
$$

and

$$
\frac{d y}{d t}=V \sin \beta .
$$

Obviously, skiers decide when to start a new turn based not on the time elapsed since the start of their run, but on the current circumstances of the run. One of the key parameters taken by them into consideration is the current angle of traverse. Hence, one may consider using as an independent variable not $t$ but $\beta$. In what follows, we deal only with highly repetitive turns, where the turn initiation and completion occur at exactly the same values of the angle of traverse, $\pm \beta_{\mathrm{m}}$. This makes the use of $\beta$ 
as an independent variable particularly advantageous. The substitution can be done using equation (11) and yields the system of three equations,

$$
\begin{gathered}
\frac{d V}{d \beta}=\frac{V}{g} \frac{G}{F}, \\
\frac{d x}{d \beta}=\frac{V^{2}}{g} \frac{\cos \beta}{F},
\end{gathered}
$$

and

$$
\frac{d y}{d \beta}=\frac{V^{2}}{g} \frac{\sin \beta}{F}
$$

where

$$
G=\sin \alpha \cos \beta-\cos \alpha \sum_{i=l, r} A_{N}^{(i)}\left|\sin \gamma^{(i)}\right| \tan \Phi^{(i)}
$$

and

$$
F=-\sin \alpha \sin \beta+\cos \alpha \sum_{i=l, r} A_{N}^{(i)} \operatorname{sgn} \gamma^{(i)} \cos \gamma^{(i)} \tan \Phi^{(i)}
$$

A small disadvantage of this approach is that a ski run cannot be computed continuously but each individual turn has to be treated separately.

During left turns $\beta$ increases from $-\beta_{\mathrm{m}}$ to $+\beta_{\mathrm{m}}$, whereas during right turns it decreases from $+\beta_{\mathrm{m}}$ to $-\beta_{\mathrm{m}}$. At $\beta= \pm \beta_{\mathrm{m}}$ the skier trajectory has an inflection point that separates consecutive turns. The values of $V, x$, and $y$ found at the end of the previous turn become the initial conditions for the next-turn computations. Following the terminology of skiing we will refer to the matching point as a point of transition between turns.

One can see that in addition to $V, x$ and $y$, equations (14)-(16) involve five more unknown functions, $A_{N}^{(l)}, \gamma^{(i)}$, and $\Phi^{(i)}$. These are not fully defined by laws of mechanics but depend on skills and preferences of individual skiers. In this context, they can be called control parameters. Here we simplify the problem by treating them as functions of $\beta$. That is $A_{N}^{(l)}=A_{N}^{(l)}\left(\beta, p_{A}\right), \gamma^{(i)}=\gamma^{(i)}\left(\beta, p_{\gamma}\right)$, and $\Phi^{(i)}=\Phi^{(i)}\left(\beta, p_{\Phi}\right)$, where we include the possibility of involving free parameters $p_{k}$, which can be helpful in identifying more suitable options from a whole family of functions.

In a wedge turn, the wedge angle, $\gamma_{\mathrm{w}}=\gamma^{(r)}-\gamma^{(l)}$, is kept approximately invariant throughout the turn. Because of the different signs of $\gamma^{(r)}$ and $\gamma^{(l)}$ the skis are generally trying to turn in the opposite directions (see equation 11). The turning power of each ski scales as $A_{N}^{(i)} \cos \gamma^{(i)} \tan \Phi^{(i)}$ and the skier turns in the direction dictated by the ski with higher turning power. As to the braking action, both skis act in unison.

According to the study by Sahashi and Ichino (1998), the angle of attack of the left ski during the right turn is close to $-\gamma_{\mathrm{w}}$ and it is close to zero during the left turn, with a quick transition from one value to another near the transition point $\beta= \pm \beta_{\mathrm{m}}$. 
Similarly, the angle of attack of the right ski during the left turn is close to $+\gamma_{\mathrm{w}}$ and it is close to zero during the right turn. Hence, we select the following simple model,

$$
\gamma^{(l)}=-A_{\gamma}^{(l)}(\beta) \gamma_{\mathrm{w}}, \quad \gamma^{(r)}=A_{\gamma}^{(r)}(\beta) \gamma_{\mathrm{w}}
$$

where $A_{\gamma}^{(r)}(\beta)+A_{\gamma}^{(l)}(\beta)=1$ and

$$
A_{\gamma}^{(l)}(\beta)= \begin{cases}\frac{1}{2}-\frac{1}{2}\left(1-\left(\frac{\beta}{\beta_{\mathrm{m}}}\right)^{2}\right)^{1 / 2} & \text { for left turns } \\ \frac{1}{2}+\frac{1}{2}\left(1-\left(\frac{\beta}{\beta_{\mathrm{m}}}\right)^{2}\right)^{1 / 2} & \text { for right turns }\end{cases}
$$

According to this model, at the transition point $\gamma^{(l)}=\gamma^{(r)}=\gamma_{\mathrm{w}} / 2$ and hence the wedge is symmetric with respect to the direction of motion. The graph of function (20) is shown in the left panel of figure 3 . According to this equation, $d \gamma^{(i)} / d \beta$ diverges at $\beta= \pm \beta_{m}$. However, on can show that $d \gamma^{(i)} / d t$ remains finite.

Sahashi and Ichino (1998) did not measure the CM position and did not provide any information on the ski inclination and edging. According to our own naked-eye observations of skiers performing wedge turns, during most of the turn the inside ski is almost flat on the snow. This makes sense as it helps to minimise the tendency of the inside ski to turn in the opposite direction to the dominant outside ski. Moreover, during the turn both skis rotate in exactly the same way. The rotation of the outside ski is achieved via the torque due to the snow reaction force, provided the front section of the ski is loaded more than the tail section. If the inside ski was on its outside (relative to the turn arc) edge, the same mechanism would be forcing this ski to rotate in the opposite direction to the outside ski. For a flat inside ski, this mechanism is deactivated, and it can be rotated in the same direction as the outside ski via torsional leg action. (In fact, if the inside ski was put on its outside edge, then the snow reaction torque would help to achieve the required rotation of this ski.) Hence we adopt the following model for the ski inclination

$$
\Phi^{(l)}=A_{\Phi}^{(l)}(\beta) \Phi_{\mathrm{m}}, \quad \Phi^{(r)}=A_{\Phi}^{(r)}(\beta) \Phi_{\mathrm{m}},
$$

where $A_{\Phi}^{(r)}(\beta)+A_{\Phi}^{(l)}(\beta)=1$ and $A_{\Phi}^{(l)}(\beta)$ exhibits a similar behaviour to $A_{\gamma}^{(l)}(\beta)$. In fact, for simplicity we will assume that

$$
A_{\Phi}^{(l)}(\beta)=A_{\gamma}^{(l)}(\beta)
$$

Hence, at the transition point, $\Phi^{(l)}=\Phi^{(r)}=\Phi_{\mathrm{m}} / 2$.

The length of the front section of a $180 \mathrm{~cm}$ long ski is about one meter, and distance from the feet to the CM of a fully extended $180 \mathrm{~cm}$ tall skier is about one 

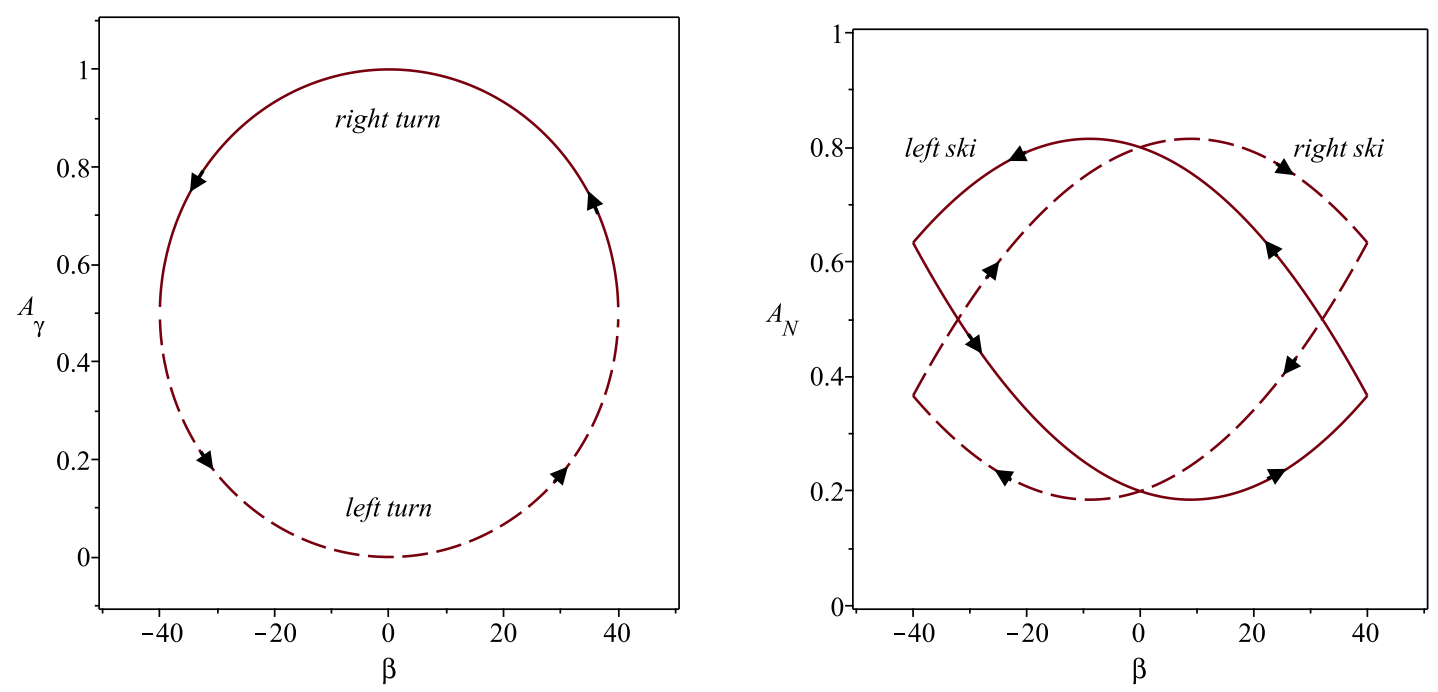

Figure 3. Left panel: The angle of attack function $A_{\gamma}^{(l)}(\beta)$ (left ski) as determined by equation (20). The arrows indicate the direction of evolution through turns. Right panel: The load distribution functions $A_{N}^{(l)}$ (solid line) and $A_{N}^{(r)}$ (dash line) as determined by equation (26). The arrows show the direction of evolution through turns. In right turns $\beta$ decreases and in left turns it increases.

meter. For a configuration where the ski tips contact each other, this implies $\Phi_{\mathrm{m}} \simeq \gamma_{\mathrm{w}}$. In reality the tips are kept apart, by at least $10 \mathrm{~cm}$, and hence

$$
\Phi_{\mathrm{m}}=\kappa \gamma_{\mathrm{w}}
$$

where $\kappa \gtrsim 1$.

At the turning points $d \beta / d t=0$ and equation (11) implies

$$
A_{N}^{(l)}=\left\{\begin{array}{ll}
A_{m} & \text { for } \quad \beta=\beta_{m} \\
1-A_{m} & \text { for } \quad \beta=-\beta_{m}
\end{array},\right.
$$

where

$$
A_{m}=\frac{1}{2}\left(1-\frac{\tan \alpha \sin \beta_{m}}{\cos \frac{\gamma_{m}}{2} \tan \frac{\Phi_{m}}{2}}\right)
$$


Using quadratic polynomials to describe $A_{N}^{(l)}(\beta)$ for left and right turns, we obtain

$$
A_{N}^{(l)}(\beta)= \begin{cases}-\left(\frac{1}{2}-A_{0}\right)\left(\frac{\beta}{\beta_{m}}\right)^{2}-\left(\frac{1}{2}-A_{m}\right)\left(\frac{\beta}{\beta_{m}}\right)+1-A_{0} & \text { for left turns } \\ \left(\frac{1}{2}-A_{0}\right)\left(\frac{\beta}{\beta_{m}}\right)^{2}-\left(\frac{1}{2}-A_{m}\right)\left(\frac{\beta}{\beta_{m}}\right)+A_{0} & \text { for right turns }\end{cases}
$$

where $A_{0}$ is the loading fraction of a dominant ski at the fall line $(\beta=0)$.

\section{Results}

The ski slope used in the experimental study by Sahashi and Ichino (1998) had the inclination $\alpha=7^{\circ}$. For wedge turns, they only present data for two consecutive turns of a single run. Based on the data, we can fix the turning point of the run to $\beta_{\mathrm{m}}=40^{\circ}$ and the wedge angle to $\gamma_{\mathrm{w}}=20^{\circ}$. The experimental data are presented in the form of plots which show the trajectory of skis and the variation of some key parameters with the distance down the fall line. These are the angle of traverse for the mid point of the ski wedge, the angles of attack for both the left and the right skis, the speed of the mid point, "the curvature radius of ski track", and the effective coefficient of snow friction. The effective coefficient of friction is obtained on the basis of the observed acceleration of the mid point and the model where it is attributed to the competition between the component of the gravity force along the direction of motion and the Coulomb friction. The way the radius of curvature is calculated is not described. Given the fact that in the wedge turn, skis leave a rather wide trail, this introduces a significant degree of uncertainty about this parameter.

Given our selection of $\beta$ as an independent variable, the computations of the whole ski run split into computations of individual turns. During left turns, $\beta$ increases from $-\beta_{\mathrm{m}}$ to $+\beta_{\mathrm{m}}$ and during the right turns it decreases from $+\beta_{\mathrm{m}}$ to $-\beta_{\mathrm{m}}$. Because $F\left( \pm \beta_{\mathrm{m}}\right)=0$, the integrated equations (14)-(16) are singular at the turning points. In order to avoid the singularity, the numerical integrations of these equations begins and terminates at $\beta= \pm 0.9999 \beta_{\mathrm{m}}$. The parameters found at the end of the previous turn determine the initial conditions for the next turn. The initial conditions for the whole run are $\beta=-0.9999 \beta_{\mathrm{m}}, V=V_{0}, x=0$, and $y=0$. In the experimental data, the run speed is more or less constant and we chose its typical value as the initial speed $V_{0}=3 \mathrm{~m} / \mathrm{s}$.

With $\beta_{\mathrm{m}}, \gamma_{\mathrm{w}}$, and $V_{0}$ immediately determined by the experimental data, the only other two model parameters which remain free are the degree of loading of the dominant ski at the fall line $A_{0}$ and the ski inclination factor $\kappa$. The realistic ranges for this parameters are $A_{0} \in(0.5,1)$ and $\kappa \in(1,2)$. Hence, we explored this section of the parameter space, looking for a reasonable fit to the remaining experimental data. As a first step, we selected $A_{0}=0.8$ and varied $\kappa$ until there was no strong systematic variation of the speed $V$ within the first 15 meters of the run. Then we tuned $\kappa$ a little bit more so that the distance between two points corresponding to 
the same turn phase was the same as in the experimental plot, $\approx 4.5$ meters, which was achieved at $\kappa=1.67$. This value of $\kappa$ corresponds to the peak ski inclination angle $\Phi_{\mathrm{m}}=34^{\circ}$. Visually, this value is consistent with the angle between skies legs as seen in figure 1 in Sahashi and Ichino (1998).

Figure 4 shows the variation of the same parameters as in figure 3 in Sahashi and Ichino (1998) for the model with $A_{0}=0.8$ and $\kappa=1.67$. In the context of this study, the most important of these parameters is the effective coefficient of friction, defined as

$$
\mu_{\mathrm{eff}}=\sum_{i=l, r} A_{N}^{(i)}\left|\sin \gamma^{(i)}\right| \tan \Phi^{(i)}
$$

Using this definition, one can write equation (9) as

$$
\frac{d V}{d t}=g\left(\sin \alpha \cos \beta-\mu_{\mathrm{eff}} \cos \alpha\right)
$$

which looks exactly the same as in the model where the snow reaction force is replaced with Coulomb's friction with the coefficient $\mu_{\text {eff. }}$ Like in the experimental plots, $\mu_{\text {eff }}$ varies between 0.05 and 0.2 , with regions of high and low values of approximately the same size. It increases on approach to the fall line and decreases after passing it. The peak value is reached just after passing the fall line. This tendency is also seen in the experimental data, perhaps even to a larger degree.

The variation of the angle of traverse $\beta$ is similar to what is seen for the first turn in Sahashi and Ichino (1998) but the transition between the first and the second turn of the experimental run is noticeably sharper. The speed plot shows qualitatively the same evolution as in the experiment, with maxima just before the fall line around the phase where the effective coefficient of friction is about half way between its extreme values. However, the amplitude of the speed variation is approximately twice as low compared to the experimental data.

The largest difference between the model an the data is in the values of the curvature radius of the trajectory

$$
R=\left|\frac{d \beta}{d l}\right|^{-1}=V\left|\frac{d \beta}{d t}\right|^{-1},
$$

where $l$ is the distance along the trajectory. While the shape and position of the theoretical curve are very similar to what is seen in the experimental curve, we find twice as lower radii at the minima. This is somewhat in conflict with the apparent similarity between our trajectory of the CM and the ski trajectories presented in Sahashi and Ichino (1998). To illustrate this point, we scanned their plot and superimposed it on our trajectory plot. The result is shown in figure 5. One can see that theoretical and the experimental trajectories agree. In fact, the theoretical curve traces the inside ski, as indeed is expected in the case where it runs flat, and hence the CM is located right above it ( with the respect to the normal of the ski slope). Presumably, the explanation of the apparent conflict between the computed and measured curvature radii lies 

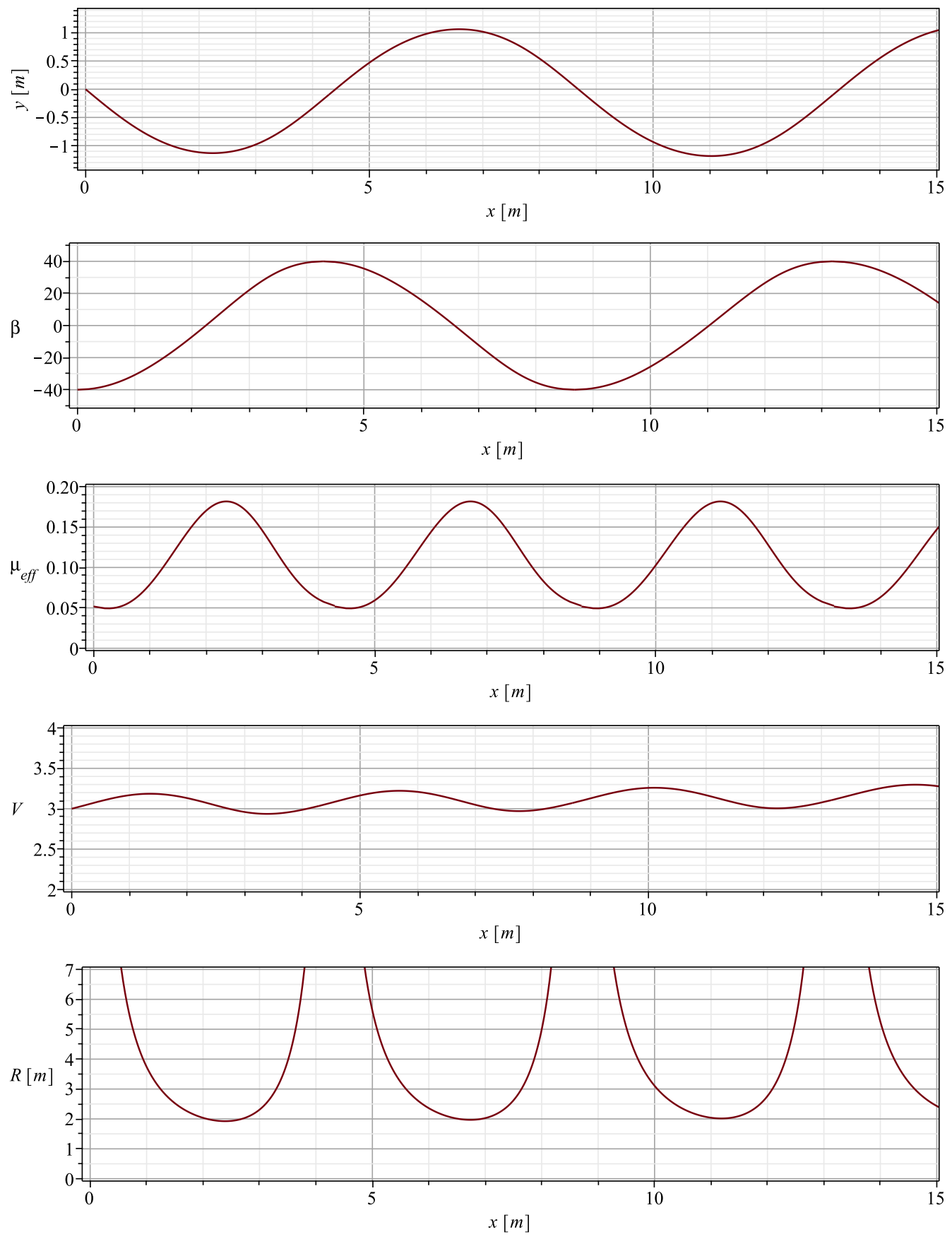

Figure 4. Variation of the key parameters throughout consecutive wedge turns in the model with $A_{0}=0.8$ and $\kappa=1.67$. 


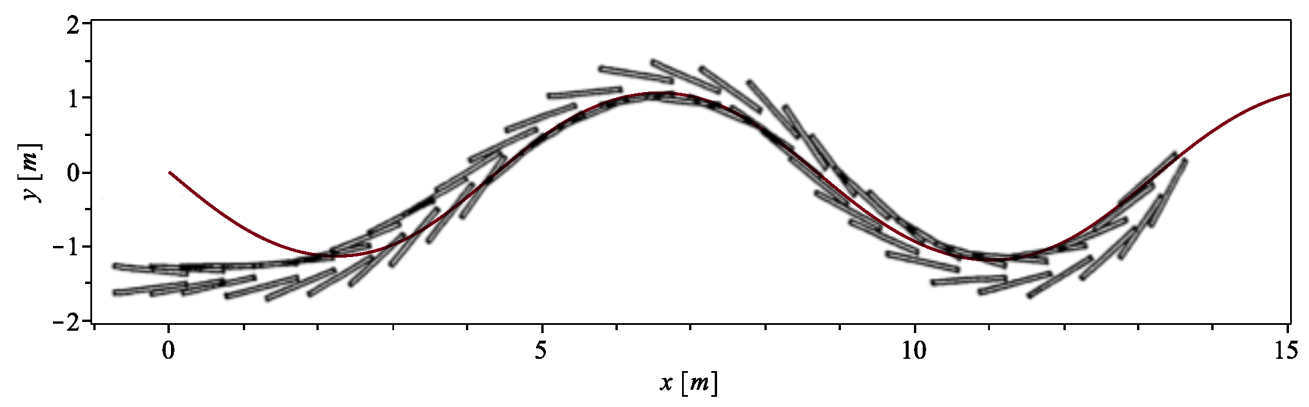

Figure 5. Superposition of the CM trajectory in the model with $A_{0}=0.8$ and $\kappa=1.67$, and the skis trajectories from Sahashi and Ichino (1998).

in the difference between their definitions. Unfortunately, Sahashi and Ichino (1998) do not describe their procedure for measuring $R$.

Overall, the computed run is quite similar to the run studied by Sahashi and Ichino (1998). Without tabulated data, we cannot do a proper fitting of the theoretical model. Moreover, the experimental data describe only two turns, which are not identical, and hence the outcome of such a fitting would not provide any useful statistical information. Hence we opted not to proceed in this direction. Instead, we simply repeated our procedure with somewhat different values of $A_{0}$ to see if this would lead to a noticeably different outcome. This way we have found that for a higher $A_{0}$ a similar outcome can be obtained with lower value of $\kappa$, and the other way around. For example, the solutions corresponding to $A_{0}=0.7$ and $\kappa=1.78$, and $A_{0}=0.9$ and $\kappa=1.57$ are not much different from the reference solution described above.

We finish this section, by considering a simpler manoeuvre involving wedged skis that can also be used for experimental verification of our model. Namely we analyse the motion down the fall line. In this case, $\beta=0, A_{N}^{(l)}=A_{N}^{(r)}=1 / 2$, $\gamma^{(l)}=\gamma^{(r)}=\gamma_{\mathrm{w}} / 2, \Phi^{(l)}=\Phi^{(r)}=\Phi_{\mathrm{m}} / 2$, and equation (9) reads

$$
\frac{1}{g} \frac{d V}{d t}=\sin \alpha-\cos \alpha \sin \left(\frac{\gamma_{\mathrm{w}}}{2}\right) \tan \left(\frac{\Phi_{\mathrm{m}}}{2}\right) .
$$

For travelling with constant speed, this equation implies

$$
\tan \alpha=\sin \left(\frac{\gamma_{\mathrm{w}}}{2}\right) \tan \left(\frac{\Phi_{\mathrm{m}}}{2}\right) .
$$

For larger wedge angle, the skier will decelerate and eventually stop. For smaller angles they will speed up. Figure 6 shows how the critical wedge angle depends on the slope inclination $\alpha$ when $\Phi_{\mathrm{m}}=\gamma_{\mathrm{w}}$. One can see that it grows rapidly at small $\alpha$ and reaches the appreciable value of $\gamma_{\mathrm{w}}=33^{\circ}$ already for slopes with $\alpha=5^{\circ}$. 


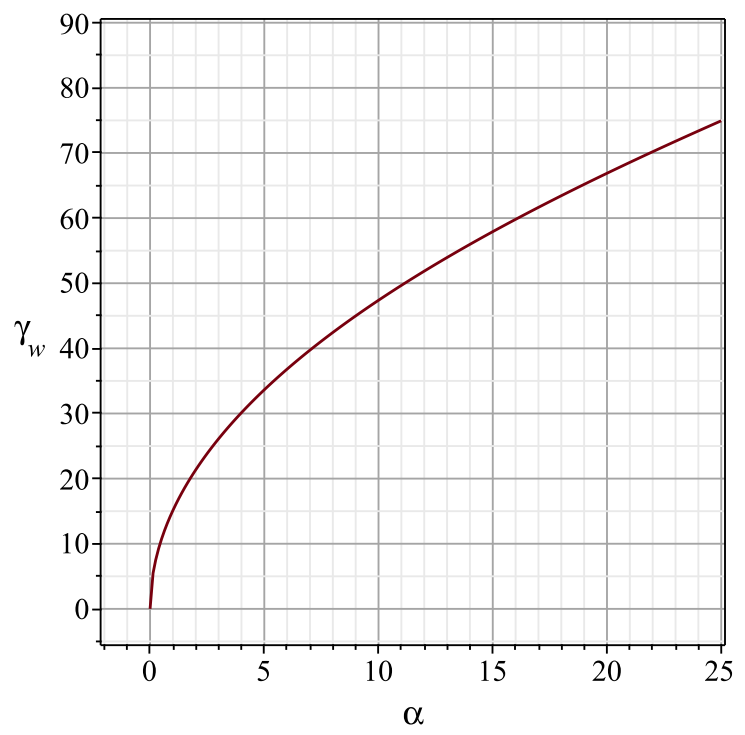

Figure 6 . Critical wedge angle above which wedge skidding down the fall line comes to stop as a function of the slope angle.

The even larger values of the wedge angle predicted for steeper slopes, with $\alpha>20^{\circ}$, require rather short skis in order to avoid their intersection.

\section{Discussion and implications}

The complex and coordinated movements of skier's body performed during skiing are designed mostly to achieve the desired interaction between their skis and snow. Indeed, the snow reaction force is the only force acting on the skier which enables the turning manoeuvre. Moreover, this interaction also allows skiers to slow down, to stop, and generally control their speed. Some contribution to the speed control is also provided by the aerodynamic drag force, but this mechanism is efficient only at very high speed. Hence, a clear understanding of the ski-snow interaction is a primary objective for the theory of skiing.

In our simplified model of the ski-snow interaction, based on the theory of machining, the key parameters determining the turning and braking forces acting on the skis are their loading, the angle of attack, and the inclination angle. The ski loading can be changed via flexion and contraction of skier's legs. These movements also allow to control ski inclination. The angle of attack can be changed via torsional action of skier's legs or indirectly via changing the load distribution along the skis. The latter allows to regulate the torsion applied to the ski by the snow. In order to preserve the leg separation against the snow reaction force that pushes wedged skis towards each other, the skis have to be constantly pushed apart by the skier's legs. The ski inclination can also be regulated via knee angulation (see figure 1). All 
these and other relevant skier actions are important and can be explored in details in more advanced studies, but they are beyond the scope of our present study. Instead, we treated the parameters governing the ski-snow interaction as control parameters and chose to specify them as functions of the turn phase (the angle of traverse). This choice is convenient for highly repetitive ski runs (periodic or quasi-periodic solutions), which are most suitable for studying the turn dynamics.

Our main task was to check whether the theory of snow machining can yield turns that are similar to the wedge turns performed by actual skiers on actual ski slopes and explain the abnormally high and variable coefficient of friction that were indirectly measured in the experimental study of such turns by Sahashi and Ichino (1998). Unfortunately, the study gives no information on the ski loading and inclination, and only rather limited graphic information on the angle of attack. Hence, we specified the control functions using video recordings of wedge turns available on youtube and our personal skiing experience. We admit that the videos reveal significant stylistic variety and hence our choice is far from being unique.

In order to simplify the comparison between the model and the experiment, we introduced only two free parameters. Although this reduced the flexibility of our model, we still succeeded in reproducing the wedge turns studied in Sahashi and Ichino (1998) quite closely. In particular, the evolution of the effective coefficient of friction with the turn phase was similar to the one observed in the experiment, including the abnormally high values near the peak. Although this is very encouraging, further investigation is required before we can make a firm conclusion. In particular, future field experiments should aim at measuring more turn parameters and provide detailed information on loading and edging of skis, as well as their angle of attack.

One of the obvious difficulties in measuring of ski turn parameters is the constant change of skier's position relative to the video-recording equipment. This, requires several cameras capturing the skier at different view angles during the whole run and a rather sophisticated software algorithm in order to reconstruct the positions of the skier and their skis. Moreover, a skier is not a robot and hence their every individual turn is somewhat different from their other turns. Various possible inconsistencies of terrain also contribute to the inconsistency of turns. Therefore, a statistical data analysis has to be done as well, which requires big data sets including many turns. Hence a simpler experiment, where possible, would be very useful. At the end of the previous section, we proposed such an experiment. It involves skiing down the fall line, while keeping the skis is a wedge configuration. In this manoeuvre, the skier is not required to repeat their movements turn after turn, but has to focus on keeping their position, and the position of their skis, unchanged, which is a simpler task. This motion is described by just one simple differential equation, which can be integrated analytically (see equation 30). Although this experiment cannot be used for verification of the theoretical prediction concerning the turning action of a skidded ski, it is quite suitable for investigation of its braking action.

The simplified snow machining model used in this study treats the ski as a flat 
plank with straight edge. In reality, most modern skis are shaped and their edge shows noticeable deviations from a straight line not only at the ski tip and tail but also in the mid-section. The shape is designed mostly to facilitate carving but it must have some effect on the ski as a snow machining tool. It would be interesting to find out what it is. As a ski skids (side-slips), it also bends, and hence the angle of attack becomes a function of the position along the ski edge (e.g. Lieu \& Mote, 1985; Reid, 2010). For turns with large mean angle of attack, like the wedge turn, this is likely to be a secondary effect. For turns with small angle of attack, approaching pure carving, the ski bending may become crucial for determining its braking power. Even a relatively small angle of attack at the skidding front section of the ski may be sufficient for the snow cutting force originated at this section to dominate Coulomb's friction. Further investigation involving much more complicated equations and computer simulations is needed to clarify this effect. Even if it is noticeable and hence important, for example to ski manufacturers, our simpler model will still be useful to wider skiing community as an educational tool.

\section{Conclusion}

In this paper we described the first attempt to model consecutive wedge turns using a simplified theory of snow machining. The results are at least in semi-quantitative agreement with the experimental data obtained by Sahashi and Ichino (1998) and hence encouraging. In particular, the snow machining mechanism allows to explain the abnormally high and dependent on the turn phase values of the friction coefficient found in that field study. However, more advanced experiments, providing statisti-

cally sufficient information for a larger set of parameters, are required to make further advances in this area.

\section{References}

Brown, C. (2009). Modeling edge-snow interactions using machining theory. In E. Müller, L. S., \& T. Stöggl (Eds.), Science and skiing iv (p. 175-182). Maidenhead, UK: Meyer \& Meyer Sport.

Kaps, P., Nachbauer, W., \& Mössner, M. (1996). Determination of kinetic friction and drag area in alpine skiing. In C. Mote, R. Johnson, W. Hauser, \& P. Schaff (Eds.), Skiing trauma and safety: Tenth volume (p. 165-177). West Conshohocken, PA: ASTM International.

Komissarov, S. (2020). Snow machining and side slipping in alpine skiing. (SportRxiv. https://doi:10.31236/osf.io/p45br)

Lieu, D., \& Mote, C. (1984). Experiments in the machining of ice at negative rake angles. Journal of Glaciology, 30, 77-81.

Lieu, D., \& Mote, C. (1985). Mechanics of the turning snow ski. In R. Johnson \& C. Mote (Eds.), Skiing trauma and safety: Tenth volume (p. 117-140). Philadelphia: ASTM International. 
Merchant, M. (1945). Mechanics of the metal cutting process. i. orthogonal cutting and a type 2 chip. J. Appl. Phys., 16, 267-275.

Reid, R. (2010). A kinematic and kinetic study of alpine skiing technique in slalom (PhD dissertation, Norwegian School of Sport Sciences). Retrieved from http://hdl.handle.net/11250/171325

Sahashi, T., \& Ichino, S. (1998). Coefficient of kinetic friction of snow skis during turning descents. Japanese Journal of Applied Physics, 37, 720-727.

Tada, N., \& Hirano, Y. (1999). Simulation of a turning ski using ice cutting data. Sports Engineering, 2(1), 55-64.

Tada, N., \& Hirano, Y. (2002). In search of the mechanics of a turning alpine ski using snow cutting force measurements. Sports Engineering, 5, 15-22.

Tada, N., \& Kobayashi, T. (2005). Measurement of snow cutting forces for analysis and design of a snow ski. Journal of Ski Science, 3, 11-21. 